\title{
Brief Communication: Chronic Undernutrition is Associated with Higher Mucosal Antibody Levels Among Ariaal Infants of Northern Kenya
}

\author{
Elizabeth M. Miller ${ }^{1 *}$ and Daniel S. McConnell ${ }^{2}$ \\ ${ }^{1}$ Department of Anthropology, Northwestern University, Evanston, Illinois 60208 \\ ${ }^{2}$ Department of Epidemiology, School of Public Health, University of Michigan, Ann Arbor, Michigan 48109
}

\author{
KEY WORDS nutrition; growth; immune function; immunoglobulin A; Kenya
}

\begin{abstract}
The immune activation that occurs with infection diverts energy from growth and can contribute to poor nutritional outcomes in developing infants and children. This study investigates the association between salivary immunoglobulin A (IgA) levels and growth outcomes among Ariaal infants of northern Kenya. The Ariaal are a group of settled northern Kenyan pastoralists who are under considerable nutritional stress. Two hundred and thirty-nine breastfeeding Ariaal infants were recruited into the study and underwent anthropometric measurement and saliva collection, with mothers providing individual and household characteristics for them via questionnaire. Infant saliva samples were analyzed with an ELISA for IgA in the United States. Infant anthropometric measurements were converted to height-for-age $z$-scores (HAZ) using
\end{abstract}

Infection during infancy and childhood takes a large toll on infant growth (Stephensen, 1999), reflecting life history trade-offs between growth and maintenance, specifically immune function (Lochmiller and Deerenberg, 2000). While short-term immune response to infection is beneficial to the individual by protecting the body from death by infection, long-term immune up-regulation can produce significant costs to individuals' growth, reproduction, and ultimately their fitness. Long-term up-regulation of immune function can occur through exposure to high pathogen environments and can potentially trade-off with growth, particularly in resource-poor populations. Recent work in populations living in high pathogen environments has found trade-offs between biomarkers of immune function and indicators of growth, such as the relationship between high C-reactive protein and slow growth among Tsimane' children (McDade et al., 2008) and high IgE and shorter stature among Shuar children (Blackwell et al., 2010). The trade-offs between growth and immune function are potentially more robust among infants, both because their immune systems are naive and constantly adapting to the surrounding pathogen ecology and because they experience high growth rates.

This study investigates the relationship between mucosal immunity and chronic undernutrition among Ariaal infants of northern Kenya. We predict that higher salivary immunoglobulin A (IgA) concentration, a biomarker of mucosal immunity, will be associated with lower height-for-age $z$-scores in Ariaal infants compared to a reference population, representing a long-term trade-off between growth and immune function. This the WHO Child Growth Standards. Based on multivariate models performed in SAS 9.2 two main results emerge: 1) low HAZ, an indicator of chronic undernutrition, was significantly associated with higher IgA concentration $(\beta=-0.12, P=0.050)$ and 2$)$ boys had significantly higher IgA levels than girls $(\beta=0.25, P=$ $0.039)$. Although there was not a significant interactive effect between HAZ and sex, the two variables confound each other, with boys having significantly lower HAZ values than girls do. In addition, maternal breastmilk IgA was significantly associated with infant salivary IgA, indicating that maternal effects play a role in infant IgA development. Future research will unravel the three-way association between sex, stunting, and immune function in the Ariaal community. Am J Phys Anthropol 149:136-141, 2012. @ 2012 Wiley Periodicals, Inc.

model is adjusted for infant characteristics such as age, sex and fat, livestock ownership (a measure of socioeconomic status), and village to account for potential household and community differences. The model also includes maternal BMI and breastmilk IgA concentration in order to control for maternal effects of nutritional status and passive immune transfer.

\section{PROXIMATE MECHANISMS OF MUCOSAL IMMUNE SYSTEM DEVELOPMENT}

The development and adaptation of the mucosal immune system to the local disease ecology is a critical process during infancy (Husband and Gleeson, 1996). The mucosal immune system is dominated by the secretory form of immunoglobulin A (IgA or sIgA in secretory form), an adaptive immune biomarker found in abundance in the gastrointestinal tract, the respiratory tract, the urogenital tract, the eyes, and in breastmilk. The

\footnotetext{
Grant sponsor: NSF DDIG; Grant numbers: BCS-0750779. Grant sponsor: Leakey Foundation General Research Grant.

*Correspondence to: Elizabeth Miller, Department of Anthropology, 1810 Hinman Ave. Evanston, IL 60208.

E-mail: elizabeth.miller2@northwestern.edu
}

Received 13 December 2011; accepted 22 May 2012

DOI 10.1002/ajpa.22108

Published online 29 June 2012 in Wiley Online Library (wileyonlinelibrary.com). 
IgA in breastmilk contains immunological memory of pathogens that the mother, and by extension the infant, experience in their environment. The IgA passed to infants via breastmilk protects infants from some of the lethal and nonlethal energetic costs of disease exposure. Despite buffering by maternal breastmilk, endogenous immune development in infants trades off with growth, particularly in high pathogen environments.

At birth, infants do not produce detectable levels of IgA. By the time they are a few weeks old, however, they have started to produce their own IgA, albeit at levels that are considerably lower than adults (Fitzsimmons et al., 1994). Infants reach adult levels of IgA in the mucosa during childhood and maintain these levels until old age (Cripps et al., 1991; Fujihashi and Kiyono, 2009). Previous research has found that IgA concentration fluctuates with nutritional status (see Chandra, 1992; McDade, 2005 for reviews). In fact, IgA levels are suppressed in infants and children who are acutely malnourished; that is, children with low weight-for-height or weight-for-age (Cripps et al., 1991; McGee and McMurray, 1988). This information, along with the large volume of IgA produced every day by the human body $(66 \mathrm{mg} / \mathrm{kg}$ body weight; Conley and Delacroix, 1987) demonstrates that IgA production could potentially trade-off strongly with long-term growth outcomes in infants and children.

\section{FIELD SITE}

The Ariaal people reside on and around the south incline of Marsabit Mountain in Marsabit District, Kenya. They have very strong ties to the Samburu and Rendille pastoralist groups, sharing cultural features with both (Fratkin, 1998). The Ariaal in this study are settled, cattle-keeping pastoralists who also rely on subsistence agriculture and market goods for their nutritional base. Although settlement may have broadened the available food base, it brought few to no improvements in nutritional status or health to the Ariaal people (Fratkin and Roth, 2004).

The Ariaal population experiences considerable nutritional stress. Men (Campbell et al., 2003), women (ShellDuncan and Yung, 2004), and children (Nathan et al., 1996) are undernourished at all ages. This is partially attributed to lower levels of milk protein available to settled individuals and poor-quality replacement diets such as maize meal (Fujita et al., 2004). Micronutrient deficiency is a concern within this population; for example, vitamin A deficiency is endemic among Ariaal women (Fujita et al., 2009). Both reproductive women (Miller, 2010) and children (Nathan et al., 1996; Shell-Duncan and McDade, 2005; Wander et al., 2009) experience relatively high rates of clinical anemia. Finally, research indicates that health is a major concern for the Ariaal people. One study found that $15.6 \%$ of children had elevated C-reactive protein levels, indicating a recent infection (Shell-Duncan and McDade, 2005). In this ecology, Ariaal infants are at risk of undernutrition and infectious disease, making trade-offs between immune function and growth more apparent.

\section{METHODS}

This study was performed with approval from the Institutional Review Board of the University of Michigan (Project number HUM00017927) and the Kenyatta National Hospital Ethical Review Committee. Permis- sion was also obtained from the District Commissioner of Marsabit District and the chiefs of Karare, Parkishon, and Kituruni to do research in the area.

\section{Data collection}

Fieldwork took place in Marsabit District, Kenya, between November 2008 and January 2009 in the villages of Karare, Parkishon, and Kituruni. Two hundred and fifty-one breastfeeding mothers and infants were recruited into the study; because of missing data and the exclusion of samples that did not meet quality or quantity requirements, the final sample size for this study was 239 mother-infant pairs. Mothers and infants participated in anthropometric measurement, breastmilk and saliva collection, and a lengthy questionnaire.

Standing height was taken for mothers and recumbent length was taken for infants. Mothers were weighed separately and with infants on a digital scale; infant weight was calculated by subtracting maternal weight from their combined weight. The weight of women who were wearing traditional jewelry was adjusted downward by $2.2 \mathrm{~kg}$, the mean weight of five traditional necklaces (s.d. $=1.0 \mathrm{~kg}$ ). Mid upper arm circumference was measured and triceps skinfold was taken three times and averaged for both mothers and infants; further skinfold measurements were not taken out of sensitivity to mothers' wishes.

Women and their infants were asked to refrain from breastfeeding for at least 1 hour prior to breastmilk and saliva collection. This assured that breastmilk was uniformly foremilk and allowed milk to clear the infant's mouth before saliva collection. Women expressed $2 \mathrm{~mL}$ milk by hand. Full breast expression was not acceptable to the participating women so therefore milk volume was not measured. Whole infant saliva was collected using disposable pipettes; the volume collected was typically less than $1 \mathrm{~mL}$. Whole samples were frozen in liquid nitrogen, shipped in dry ice to the United States, and was stored in $-80^{\circ} \mathrm{C}$ freezers.

Mothers also completed an oral questionnaire, translated into Samburu by two female research assistants. This questionnaire covered, among other things, maternal reproductive history and characteristics, infant characteristics, household socioeconomic status and composition, hygiene practices, and infant feeding practices.

\section{Laboratory methods}

The IgA assay used in this study was developed at the Clinical Ligand Assay Satellite Service (CLASS) laboratory at the University of Michigan. In brief, infant saliva and maternal breastmilk were assayed for IgA using a sandwich ELISA developed by the authors (Miller and McConnell, 2011). This assay was designed to capture most forms of IgA within mucosal fluids, including the dominant secretory form ( $\mathrm{sIgA}$ ) as well as the monomeric (IgA) and polymeric (pIgA) forms that are also active within mucosal fluids (Conley and Delacroix 1987). High and low controls were included with all assays, and between-assay coefficients of variation of controls were 9.1 and $9.4 \%$ for IgA concentrations of $341.8 \mathrm{ng} / \mathrm{mL}$ and $132.5 \mathrm{ng} / \mathrm{mL}$, respectively.

\section{Statistical methods}

Infant height and ages were converted into height-for age $z$-scores (HAZ) based on the WHO Child Growth ref- 
TABLE 1. Means and standard deviations or percentages for selected Ariaal infant and maternal characteristics, combined and by infant sex

\begin{tabular}{|c|c|c|c|}
\hline & Combined $(n=239)$ & Boys $(n=111)$ & Girls $(n=128)$ \\
\hline Salivary $\operatorname{IgA}(\mu \mathrm{g} / \mathrm{mL})^{\mathrm{a}}$ & $89.4 \pm 116.3$ & $106.7 \pm 157.5$ & $74.3 \pm 58.2$ \\
\hline Age (months) & $10.6 \pm 6.0$ & $10.4 \pm 6.1$ & $10.7 \pm 6.0$ \\
\hline Height $(\mathrm{cm})$ & $69.7 \pm 7.7$ & $69.9 \pm 7.7$ & $69.6 \pm 7.6$ \\
\hline Weight (kg) & $7.6 \pm 1.6$ & $7.8 \pm 1.6$ & $7.5 \pm 1.5$ \\
\hline Mid-upper arm circumference $(\mathrm{cm})$ & $14.2 \pm 1.3$ & $14.3 \pm 1.3$ & $14.2 \pm 1.3$ \\
\hline Mean triceps skinfold (mm) & $9.4 \pm 2.5$ & $9.4 \pm 2.5$ & $9.4 \pm 2.6$ \\
\hline Body mass index $\left(\mathrm{kg} / \mathrm{m}^{2}\right)$ & $15.7 \pm 1.7$ & $15.9 \pm 1.7$ & $15.5 \pm 1.6$ \\
\hline Height-for-age z-score (HAZ) ${ }^{a}$ & $-0.82 \pm 1.4$ & $-1.0 \pm 1.5$ & $-0.62 \pm 1.2$ \\
\hline$\%$ stunted $(\mathrm{HAZ}<-2.0)^{\mathrm{a}}$ & $17.6 \%$ & $26.1 \%$ & $10.2 \%$ \\
\hline Weight-for-age z-score (WAZ) & $-0.97 \pm 1.3$ & $-1.1 \pm 1.3$ & $-0.83 \pm 1.2$ \\
\hline$\%$ underweight $(\mathrm{WAZ}<-2.0)$ & $19.2 \%$ & $22.5 \%$ & $16.4 \%$ \\
\hline Weight-for-height z-score (WHZ) & $-0.70 \pm 1.3$ & $-0.77 \pm 1.4$ & $-0.64 \pm 1.3$ \\
\hline$\%$ wasted $(\mathrm{WHZ}<-2.0)$ & $15.1 \%$ & $17.1 \%$ & $13.3 \%$ \\
\hline Livestock units & $4.4 \pm 4.5$ & $4.6 \pm 5.2$ & $4.2 \pm 3.9$ \\
\hline Maternal BMI $\left(\mathrm{kg} / \mathrm{m}^{2}\right)^{\mathrm{a}}$ & $18.5 \pm 2.2$ & $18.2 \pm 2.2$ & $18.8 \pm 2.3$ \\
\hline Breastmilk IgA ( $\mu \mathrm{g} / \mathrm{mL})$ & $843.3 \pm 803.5$ & $826.7 \pm 790.9$ & $862.4 \pm 823.8$ \\
\hline$\%$ living in Karare & $51.1 \%$ & $52.3 \%$ & $50.0 \%$ \\
\hline$\%$ living in Parkishon & $27.6 \%$ & $27.0 \%$ & $28.1 \%$ \\
\hline$\%$ living in Kituruni & $21.3 \%$ & $20.7 \%$ & $21.9 \%$ \\
\hline
\end{tabular}

${ }^{\mathrm{a}} p<0.05$ significant difference between boys and girls.

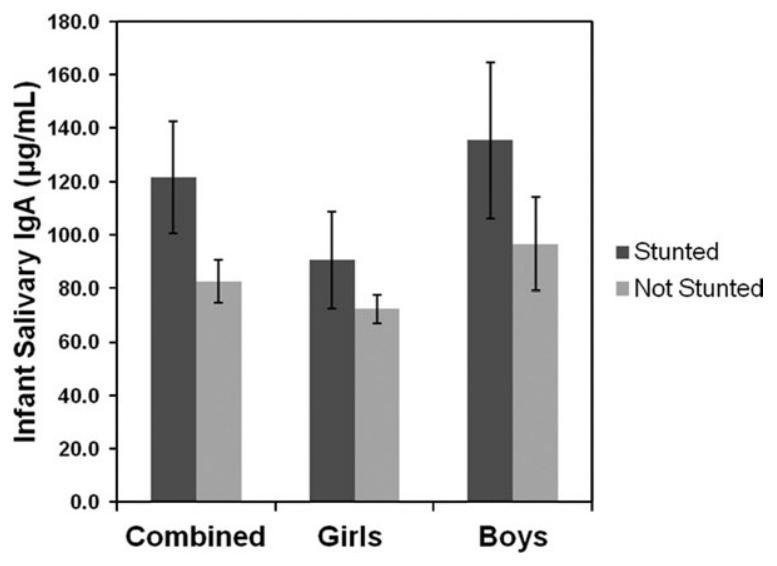

Fig. 1. The relationship between infant salivary IgA, sex, and stunting $(\mathrm{HAZ}<-2.0)$ among Ariaal infants. Error bars represent \pm 1 SEM.

erence population (WHO Multicentre Growth Reference Study Group 2006). This reference population was chosen as it better represents the growth of breastfed infants compared to CDC reference populations (de Onis et al., 2007). Infant mean triceps skinfold and mid-upper arm circumference was converted into upper arm fat area (UAFA) using equations from Frisancho (2008). To control for household wealth, total family livestock was converted into livestock units (LU), a measure that indicates the relative productivity of small and large livestock in Sub-Saharan Africa (FAO, 2009; Fratkin and Roth, 1990). In order to explore potential confounding due to differences in milk synthesis, the effect of breastmilk creamatocrit (a measure of total milk fat) on breastmilk IgA and infant salivary IgA was preliminarily examined both as a covariate and as a ratio of breastmilk IgA to fat. Ultimately, creamatocrit did not significantly affect the relationship between breastmilk IgA and infant salivary IgA and was not significant as a covariate, and was therefore excluded from the final model. Descriptive statistics and multivariate linear regression were performed in SAS 9.2. Variables were checked for normality, and variables showing non-normal distributions were log-transformed; specifically, infant salivary IgA, breastmilk IgA, maternal BMI, and LU. A linear regression with infant salivary IgA as the dependent variable and $\mathrm{HAZ}$ as the main independent variable was performed using PROC REG. The model was adjusted for infant age, sex, UAFA, log breastmilk IgA, log maternal BMI, village, and log LU. In addition, an interaction term of sex*HAZ was included in the model, described in more detail below. Significance was assessed at $\alpha=0.05$.

\section{RESULTS}

Infant characteristics, combined and by sex, are found in Table 1. Boys have significantly higher salivary IgA levels and lower HAZ than girls. A significantly higher percentage of boys are stunted than girls. Despite differences in growth indicators, there is no significant difference in triceps skinfold thickness between the sexes. Because the relationship between sex and HAZ potentially had interactive effects, an interaction term of sex*HAZ was included in the regression model. The relationship between stunting, sex, and salivary IgA levels is shown in Figure 1.

Multivariate linear regression reveals that log infant IgA levels are significantly associated with HAZ. Specifically, as HAZ decreases salivary IgA levels increase. In addition, boys have significantly higher salivary IgA levels than girls. However, the interaction between sex and $\mathrm{HAZ}$ was not statistically significant. In addition, village was also a significant covariate, with infants living in Parkishon and Kituruni having lower IgA levels than infants living in Karare. Finally, mothers' log breastmilk IgA level is significantly associated with infant log salivary IgA level.

Although there is no interaction between HAZ and sex in statistical models, they covary, making them potentially confounding variables. Confounding occurs when an independent variable influences the relationship between a predictor variable and the dependent outcome variable. In epidemiological statistics, confounding is assessed by comparing the effect size $(\beta)$ of the variable 
TABLE 2. Regression coefficients and P-values for multivariate linear regression of $H A Z$ and covariates against log infant salivary IgA

\begin{tabular}{lc}
\hline$R^{2}=0.084$ & Log salivary IgA $\beta(p)$ \\
\hline Infant age & $-0.0050(0.61)$ \\
Infant sex $^{\mathrm{a}}$ & $0.25(0.039)^{\mathrm{b}}$ \\
Height-for-age z-score (HAZ) $_{\text {Sex*HAZ }}$ & $-0.12(0.050)^{\mathrm{b}}$ \\
Upper arm fat area (UAFA) $^{*}$ & $0.056(0.47)$ \\
Log breastmilk IgA & $-0.0013(0.97)$ \\
Log maternal BMI $^{\mathrm{L}}$ & $0.30(0.047)^{\mathrm{b}}$ \\
Lives in Parkishon $^{\mathrm{c}}$ & $0.31(0.50)$ \\
Lives in Kituruni $^{\mathrm{c}}$ & $-0.45(0.00070)^{\mathrm{b}}$ \\
Log total livestock units $^{\mathrm{b}}$ & $-0.44(0.0019)^{\mathrm{b}}$ \\
\hline
\end{tabular}

${ }^{\text {a }}$ Reference category is girls.

${ }^{\mathrm{b}} P<0.05$.

${ }^{\mathrm{c}}$ Reference category is Karare.

of interest in a model without the potential confounder to a model containing the potential confounder. If the effect size of the variable of interest differs by more than $10 \%$ between models, than the variable of interest is said to be confounded by the other variable (Friis and Sellers, 2009). The regression model from Table 2 was rerun without the interaction term to create the adjusted model. We had no a priori hypothesis about the direction of confounding, so both HAZ and sex were investigated as potential confounders on each other. Two crude models, one without HAZ and the other without sex were performed. The inclusion of sex in a model containing $\mathrm{HAZ}$ changes the effect size of HAZ by $14.6 \%$, indicating that sex confounds the effect of HAZ on IgA. On the other hand, the inclusion of $\mathrm{HAZ}$ in the crude model with only sex changed the effect of sex on IgA by $16.4 \%$. For both variables, the inclusion of the confounder decreased the size of the effect. This indicates that sex and HAZ confound each other, independent of their relationship with IgA.

\section{DISCUSSION}

Higher infant IgA levels were associated with lower infant HAZ, indicating that mucosal immune functioning is elevated in infants who are chronically malnourished. Unfortunately, the data are unable to distinguish between four possible explanations for this phenomenon: 1) that chronically high levels of salivary IgA levels takes energy from growth, contributing to stunting, 2) that chronically malnourished infants prioritize IgA production to protect them from pathogens that put them at higher risk for poor morbidity and mortality outcomes, 3) that higher levels of infectious disease contribute to both growth faltering and establishment of the IgA response, or 4) that IgA elevation overcompensates for nutrition-related immune depression in cellmediated immunity (Chandra, 1997; McDade, 2005). These explanations may be interrelated, forming an adaptive complex that protects undernourished infants from infectious disease mortality. Interestingly, indicators of acute malnutrition, such as weight-for-age or weight-for-height, were not significantly associated with infant IgA among Ariaal infants (analyses not shown). Future longitudinal research will determine if IgA activation plays a causal role in poor growth outcomes among Ariaal infants.

Infant sex is also implicated in the relationship between of HAZ and infant IgA. Infant sex has a signif- icant main effect on IgA, with boys having higher levels than girls. However, the effect is not interactive. That is, one sex does not show a significantly different relationship between IgA and HAZ than the other. On the other hand, sex and HAZ do confound each other, that is, one significantly affects the other in multivariate models. This effect occurs, in part, because boys are significantly more likely to be chronically malnourished than girls in this population. This corresponds to a growing body of literature that suggests that boys are more vulnerable to environmental stressors than girls, with male children exhibiting higher mortality and responsiveness to nutritional supplementation (Stinson, 1985), greater variation in programmed lipid levels (Kuzawa and Adair, 2004), and poorer nutritional status and higher C-reactive protein (DeCaro et al., 2010) compared to girls. Like these populations, the current evidence from the Ariaal suggests that male infants are more responsive to both poor nutritional status and pathogen exposure, reflected in their higher salivary IgA levels and higher levels of stunting. In line with this vulnerability, the Ariaal population as a whole may conform to the Trivers-Willard prediction of improved female offspring condition in a resource-poor population, a pattern found in other Kenyan populations (Trivers and Willard, 1973; Cronk, 2000). However, when male and female infant outcomes are stratified by good and poor maternal condition within the population, predicted Trivers-Willard outcomes are not confirmed (analyses not shown). Further research can unpack the potential social and biological reasons for this sex difference in nutritional status.

There is also a significant relationship between mothers' breastmilk IgA and infant salivary IgA. This suggests three possibilities: 1) that breastmilk immunity affects the developing infant immune system, forming an integrated immune system between mother and infant (Brandtzaeg, 2003), 2) that correlated breastmilk and infant salivary IgA concentrations reflect shared exposure to a disease ecology, or 3) that breastmilk does not clear the infant's mouth completely before saliva collection. Because infants were not breastfed for at least an hour before saliva collection, it is not likely that breastmilk remained in the mouth; however, this effect cannot be ruled out and remains a limitation of the reported result. On the other hand, previous work has established mixed evidence of an effect of breastmilk IgA on infant mucosal IgA development (Prentice, 1987; Böttcher et al., 2003; Maruyama et al., 2009; Piirainen et al., 2009). While there are no data that directly assesses the possibility of shared mother-infant disease ecology, complementary foods are a potential agent of common exposure: $87.9 \%$ of infants in the sample were fed cow milk or staple foods that may have been shared with the household. For each possible explanation breastmilk IgA should be included in statistical models of infant salivary biomarkers to control for these effects.

Finally, village is a significant predictor of infant IgA, with infants in Parkishon and Kituruni having lower levels than infants in Karare. Because of its geographical size, Karare may be more heterogeneous than the other two communities potentially increasing variation in infant immune development. It is currently unknown how these villages vary in terms of disease ecology and infant exposure; future research will explore the relationship between hygiene and animal use, local 
microbes, and immune development within this population.

Although this study found several factors that significantly predict variation in infant $\operatorname{IgA}$, the $R^{2}$ of this study is relatively low. Since the statistical model used here only accounts for $8.4 \%$ of the total variation found in infant salivary IgA, there are likely other significant predictors that play a role in explaining this system. As salivary IgA is a general indicator of the defensive abilities of the mucosal immune system rather than a direct marker of infection (Mestecky, 1993), it reacts to a wide variety of potential stressors beyond disease ecology, including mood, stress hormones, socioeconomic status, and age (Kugler et al., 1993, Evans et al., 2000). Future research should address other causes of variation in the infant salivary IgA system.

\section{ACKNOWLEDGMENTS}

This manuscript has been greatly improved by constructive reviews from the associate editor and two anonymous reviewers. The authors thank Bill Leonard, Roberto Frisancho, Thom McDade, John Mitani, Bobbi Low, and Milford Wolpoff for their helpful comments on earlier versions of this manuscript. Bettina Shell-Duncan and Masako Fujita generously introduced the first author to Northern Kenya and Ariaal/Rendille. They also thank the field assistants Korea Leala, Rapheala Leado, Selena Gambare, and Abdulai Khalifa for their dedicated, careful work. Permission for this research was granted by the Ministry of Science and Technology of the Republic of Kenya, the District Commissioner of Marsabit District, and the chiefs of Karare, Parkishon, and Kituruni. Finally, they thank the Ariaal women and infants who participated in this study.

\section{LITERATURE CITED}

Blackwell AD, Snodgrass JJ, Madimenos FC, Sugiyama LS. 2010. Life history, immune function, and intestinal helminths: trade-offs among immunoglobulin E, C-reactive protein, and growth in an Amazonian population. Am J Hum Biol 22:836848.

Bottcher MF, Jenmalm MC, Bjorksten B. 2003. Cytokine, chemokine and secretory IgA levels in human milk in relation to atopic disease and IgA production in infants. Pediatr Allergy Immunol 14:35-41.

Brandtzaeg P. 2003. Mucosal immunity: integration between mother and the breast-fed infant. Vaccine 21:3382-3388.

Campbell B, O'Rourke MT, Lipson SF. 2003. Salivary testosterone and body composition among Ariaal males. Am J Hum Biol 15:697-708.

Chandra RK. 1992. Protein-energy malnutrition and immunological responses. J Nutr 122:597-600.

Chandra RK. 1997. Nutrition and the immune system: an introduction. Am J Clin Nut 66:S460-S463.

Conley ME, Delacroix DL. 1987. Intravascular and mucosal immunoglobulin A: two separate but related systems of immune defense? Ann Intern Med 106:892-899.

Cripps AW, Gleeson M, Clancy RL. 1991. Ontogeny of the mucosal immune response in children. Adv Exp Med Biol 310:87-92.

Cronk L. 2000. Female-biased parental investment and growth performance among the Mukogodo. In: Cronk L, Chagnon N, and Irons W, editors. Adaptation and human behavior: an anthropological perspective. Hawthorne, NY: Aldine de Gruyter.

de Onis M, Garza C, Onyango AW, Borghi E. 2007. Comparison of the WHO child growth standards and the CDC 2000 growth charts. J Nutr 137:144-148.
Evans P, Der G, Ford G, Hucklebridge F, Hunt K, Lambert S. 2000. Social class, sex, and age differences in mucosal immunity in a large community sample. Brain Behav Immun 14:41-48.

FAO. 2009. Compendium of agricultural-environmental indicators (1989-91 to 2000). Rome: Food and Agriculture Organization of the United Nations.

Fitzsimmons SP, Evans MK, Pearce CL, Sheridan MJ, Wientzen R, Cole MF. 1994. Immunoglobulin A subclasses in infants' saliva and in saliva and milk from their mothers. J Pediatr 124:566-573

Fratkin E, Roth EA. 1990. Drought and economic differentiation among Ariaal pastoralists of Kenya. Hum Ecol 18:385-402

Fratkin EM. 1998. Ariaal pastoralists of Kenya: surviving drought and development in Africa's arid lands. Boston: Allyn and Bacon.

Fratkin EM, Roth EA. 2004. As pastoralists settle: social, health, and economic consequences of the pastoral sedentarization in Marsabit District, Kenya. New York: Kluwer Academic/Plenum Publishers.

Friis R, Sellers T. 2009. Epidemiology for public health practice. Sudbury, MA: Jones \& Bartlett Publishers.

Frisancho AR. 2008. Anthropometric standards: an interactive nutritional reference of body size and body composition for children and adults. Ann Arbor: University of Michigan Press.

Fujihashi K, Kiyono H. 2009. Mucosal immunosenescence: new developments and vaccines to control infectious diseases. Trends Immunol 30:334-343.

Fujita M, Brindle E, Rocha A, Shell-Duncan B, Ndemwa P, O'Connor KA. 2009. Assessment of the relative dose-response test based on serum retinol-binding protein instead of serum retinol in determining low hepatic vitamin A stores. Am J Clin Nutr 90:217-224.

Fujita M, Roth EA, Nathan MA, Fratkin E. 2004. Sedentism, seasonality, and economic status: a multivariate analysis of maternal dietary and health statuses between pastoral and agricultural Ariaal and Rendille communities in northern Kenya. Am J Phys Anthropol 123:277-291.

Husband AJ, Gleeson M. 1996. Ontogeny of mucosal immunity: environmental and behavioral influences. Brain Behav Immun 10:188-204.

Kugler J, Hess M, Haake D. 1993. What accounts for the interindividual variability of sIgA concentration in saliva? Ann NY Acad Sci 694:296-298.

Kuzawa CW, Adair LS. 2004. A supply-demand model of fetal energy sufficiency predicts lipid profiles in male but not female Filipino adolescents. Eur J Clin Nutr 58:438-448.

Lochmiller RL, Deerenberg C. 2000. Trade-offs in evolutionary immunology: just what is the cost of immunity? Oikos 88:87-98.

McDade TW. 2005. The ecologies of human immune function. Ann Rev Anthropol 34:495-521.

McDade TW, Reyes-Garcia V, Tanner S, Huanca T, Leonard WR. 2008. Maintenance versus growth: investigating the costs of immune activation among children in lowland Bolivia. Am J Phys Anthropol 136:478-484.

McGee DW, McMurray DN. 1988. Protein malnutrition reduces the IgA immune response to oral antigen by altering B-cell and suppressor T-cell functions. Immunology 64:697-702.

Mestecky J. 1993. Saliva as a manifestation of the common mucosal immune system. Ann NY Acad Sci 694:184-194.

Miller EM. 2010. Maternal hemoglobin depletion in a settled northern Kenyan pastoral population. Am J Hum Biol 22: 768-774.

Miller EM, McConnell DS. 2011. The stability of immunoglobulin A in human milk and saliva stored on filter paper at ambient temperature. Am J Hum Biol 23:823-825.

Maruyama K, Hida M, Kohgo T, Fukunaga Y. 2009. Changes in salivary and fecal secretory IgA in infants under different feeding regimens. Pediatr Int 51:342-345.

Nathan MA, Fratkin EM, Roth EA. 1996. Sedentism and child health among Rendille pastoralists of northern Kenya. Soc Sci Med 43:503-515. 
Piirainen L, Pesola J, Pesola I, Komulainen J, Vaarala O. 2009. Breastfeeding stimulates total and cow's milk-specific salivary IgA in infants. Pediatr Allergy Immunol 20:295-298.

Prentice A. 1987. Breast feeding increases concentrations of IgA in infants' urine. Arch Dis Child 62:792-795.

Shell-Duncan B, McDade T. 2005. Cultural and environmental barriers to adequate iron intake among northern Kenyan schoolchildren. Food Nutr Bull 26:39-48.

Shell-Duncan B, Yung SA. 2004. The maternal depletion transition in northern Kenya: the effects of settlement, development and disparity. Soc Sci Med 58:2485-2498.

Stephensen CB. 1999. Burden of infection on growth failure. J Nutr 129:534S-538S.
Stinson S. 1985. Sex differences in environmental sensitivity during growth and development. Am J Phys Anthropol 28:123-147.

Trivers RL, Willard DE. 1973. Natural selection of parental ability to vary sex-ratio of offspring. Science 179:90-92.

Wander K, Shell-Duncan B, McDade TW. 2009. Evaluation of iron deficiency as a nutritional adaptation to infectious disease: an evolutionary medicine perspective. Am J Hum Biol 21: 172-179.

WHO Multicentre Growth Reference Study Group. 2006. WHO child growth standards: Length/height-for-age, weight-for-age, weight-for-length, weight-for-height and body mass index-for-age: methods and development. Geneva: World Health Organization. 